Cahiers d'études italiennes

\title{
Les femmes dans la Chrysis de Piccolomini
}

Jean-Claude Ternaux

\section{OpenEdition}

\section{Journals}

Édition électronique

URL : http://journals.openedition.org/cei/75

DOI : $10.4000 /$ cei.75

ISSN : 2260-779X

\section{Éditeur}

UGA Éditions/Université Grenoble Alpes

Édition imprimée

Date de publication : 15 octobre 2011

Pagination : 69-82

ISBN : 978-2-84310-207-3

ISSN : 1770-9571

Référence électronique

Jean-Claude Ternaux, «Les femmes dans la Chrysis de Piccolomini », Cahiers d'études italiennes [En ligne], 13 | 2011, mis en ligne le 15 avril 2013, consulté le 27 mars 2021. URL : http:// journals.openedition.org/cei/75; DOI : https://doi.org/10.4000/cei.75 


\title{
LES FEMMES DANS LA CHRYSIS DE PICCOLOMINI
}

\author{
Jean-Claude Ternaux
}

Université de Reims

La Chrysis est une pièce écrite par un homme, le futur pape Pie II, pour des hommes, ses collègues de la diète des princes électeurs, qui se tient à Nuremberg, en I444. Piccolomini est alors secrétaire de la chancellerie impériale. Il s'agit d'amuser les participants, mais on verra que ce divertissement a une portée plus grave qu'il n'y paraît. Avec cette comédie, Enea Silvio tend à ses amis de la cour un miroir plus ou moins déformant où ils peuvent reconnaître la vie qu'ils mènent. Dans cette existence où le plaisir règne en maître, celui de la bouche le dispute à celui de la couche. Ces jeunes gens peuvent faire leurs les paroles de Pythias (v. I27-I28) : « [...] potus eque atque coitus / sapiunt mi $[c] h i[\ldots]^{\mathrm{I}}$ " (" [...] la boisson autant que la copulation sont ma récréation "). Dans ces conditions, il n'est pas étonnant de constater que le regard porté sur la femme soit double. Il relève d'une tradition littéraire, celle de la comédie antique, qui s'accorde avec les mentalités du temps. Mais avant de se pencher sur les modèles et sur la misogynie qui marque profondément l'œuvre, il convient d'adopter une approche dramaturgique.

\section{Les femmes dans le système des personnages et dans l'action}

Mettant en avant le personnage féminin de Chrysis, le titre constitue un trompe-l'œil. L'exactitude exigerait que l'on renomme cette comédie Chrysis et Cassina. En effet, les personnages fonctionnent par couples. Chrysis et Cassina sont deux prostituées qui dupent deux clercs d'âge mûr, Theobulus et Dyophanes, avec deux jeunes gens, Sedulius et Charinus. Alors que les

I. "Tu mi [c]hi vas tuum das, ego tibi meum / utendum [...]. " ("Tu me donnes ton vase, je me fais un devoir de te donner le mien [...]. ») 
senes ont payé le bain, ce sont les iuuenes qui jouissent de leurs faveurs, à la scène 3. La symétrie préside donc à la répartition des personnages principaux. Deux prostituées, deux vieux, deux jeunes. Un autre couple constitue une sorte de copie des tandems formés par les prostituées et leurs jeunes amisclients, Pythias, la sœur de Chrysis, et Lybiphanes, le serviteur de Sedulius. En décidant de rentrer dans la maison pour s' " échanger leurs vases ", comme ils disent métaphoriquement et fort peu galamment aux vers I5O-I5I, ils imitent l'une la sœur, l'autre le maitre. On trouve ici une situation traditionnelle de comédie où des personnages de second rang jouent, sur le mode mineur, la partition que des personnages principaux interprètent sur le mode majeur. Enfin, un dernier couple est formé par la prostituée Antiphila et Archimenides, ami de Sedulius, aussi amoureux que lui. Nous sommes donc en présence de quatre couples dont les femmes, à l'exception de Pythias, sont vénales. Toutes ont en commun un fort désir d'aller au lit. Mais à ces femmes lubriques, il faut en ajouter une, qui fait basculer la supériorité numérique du côté féminin, Canthara, du moins jusqu'à la scène I3 où l'arrivée de Congrio, le serviteur de Dyophanes, rétablit l'équilibre.

Si la maison de l'entremetteuse Canthara est centrale dans le dispositif scénique, le rôle de la maquerelle dans l'action ne l'est pas. À la scène 5, elle se borne à différer le plaisir d'Archimenides et d'Antiphila en leur disant qu'il est l'heure de satisfaire sa bouche (v. 250-25I) et la leur :
Vos istec missa facite; post repetite cum accubabitis. Iam cenare nos et bibere tempus est ${ }^{2}$.

Elle fait patienter les deux clercs, à la scène 6, en affirmant que Chrysis et Cassina vont arriver. Si, à la scène II, elle dit qu'il faut trouver deux nouveaux clients à ses filles, c'est Pythias qui, à la scène 2 , a prétexté l'arrivée du frère de Chrysis pour faire quitter l'établissement de bains aux deux gourgandines et les a ainsi soustraites aux deux vieux lubriques. Et c'est Lybiphanes qui, dans la tradition des serviteurs rusés, imagine le stratagème permettant d'éloigner les deux importuns. C'est seulement à la fin, à la scène $\mathrm{I7}$, que le rôle de la maquerelle est un peu actif, dans la mesure où elle parle fort afin de se faire entendre de Theobulus et Dyophanes qu'il faut continuer à duper.

2. Texte latin établi par J.-L. Charlet (Enea Silvio Piccolomini, Chrysis, Paris, H. Champion, coll. « Textes de la Renaissance ", 2006), traduction personnelle : "Abandonnez cela; vous vous y remettrez / quand vous serez couchés. Il est temps pour / Nous de boire et de manger. " 
Retenons que les deux prostituées sont l'objet de l'action, que les sujets en sont les deux clercs qui, dans leur quête du plaisir, rencontrent comme opposants Pythias et Lybiphanes. Comme dans la farce, l'action repose sur un mauvais tour. De ce point de vue, la Chrysis diffère quelque peu des comédies de Plaute. Pas de reconnaissance, pas de substitutions, pas de situation triangulaire, pas de passé qui remonte à la surface, pas d'intrigue compliquée et savamment orchestrée dans laquelle se perd parfois le spectateur inattentif, mais une action qui suit une ligne simple assurant le comique. Theobulus et Dyophanes se font gruger au cours de la pièce lorsque les deux femmes reçoivent leurs amants. Mieux encore, c'est le triomphe des deux prostituées qui est assuré au dénouement. À la scène I8, v. 775 et suivants, les deux dupes demandent pardon :

DYO. Audistin que ille inter se dicerent?

THEO. Vsque a principio omnia, et gaudeo.

DYO. Quid ergo dicis?

THEO. Nos malos et illas bonas.

DYO. Quid faciemus?

THEO. Reuertemur in graciam,

orabimus ueniam, supplicabimus ${ }^{3}$.

Ce triomphe, qui consiste dans le renversement total de la situation, est lui aussi farcesque. Alors qu'elles sont en faute, les deux femmes obtiennent des excuses.

De plus, on fait payer les deux benêts : ils devront donner « trois tonneaux de leur meilleur vin vieux " (v. 802) et offrir une ceinture (v. 804). Dans la guerre des sexes, ce sont les femmes qui, de loin, l'emportent. Manipulés par ces expertes en tromperie, les deux vieux naïfs, contre toute attente, conservent leur statut de cocus ignorants, alors que la réalité devrait leur faire voir leur infortune, et que, comme on le verra plus loin, ils manifestent parfois une certaine lucidité. Elles leur font prendre des vessies pour des lanternes.

Cette chute est d'autant plus grande qu'au début de la pièce, les deux personnages faisaient l'éloge de leur situation et qu'ils mettaient l'accent sur la qualité des relations qu'ils entretiennent avec les femmes. Ainsi, Dyophanes déclare-t-il (v. IO-I5) :

Nulli pulcra quam nobis gracior Venus.

Non, ut alii, stabilem uxorem ducimus,

3. DYO. «As-tu bien entendu leur conversation? "-THEO. «Tout, depuis le début, et j'en suis content. " - DYO. «Qu'en dis-tu? » - THEO. "Que la mauvaiseté est de notre côté, la bonté du leur. » - DYO. "Que ferons-nous? " - THEO. " Nous rentrerons en grâce, / en implorant leur pardon. " 
que, si stomacosa sit, habenda est tamen. Noua in dies connubia, himeneos nouos Celebramus : si placet amor, reuertimur; ubi displicet, alio flectimus iter ${ }^{4}$.

Toute la pièce montre le contraire. Vénus, qui n'est que la Vénus des carrefours, leur est hostile, la liberté affichée avec fanfaronnade est en réalité une aliénation qui va faire coucher dehors, dans l'inconfort, les deux clercs prisonniers de leur désir. La comédie met sous les yeux des spectateurs la défaite de ces deux libidineux qui étaient pourtant sûrs de passer une nuit d'amour, comme l'indique avec gourmandise Theobulus (v. 18-22) :

$$
\begin{aligned}
& \text { [...] Sed quali iam nos putas } \\
& \text { usuros nocte? Dum subagitabis tuam, } \\
& \text { egon, adherentem pectori meo meam } \\
& \text { tenens, dabo suauium et labrum mordicus } \\
& \text { stringam et niueas milies premam papillas'? }
\end{aligned}
$$

La jactance initiale cèdera la place à l'humiliation. La structure de la Chrysis repose sur le conflit entre le principe de plaisir et celui de réalité. Pensant suivre l'un, les deux hommes rencontrent l'autre. Les deux vieux clients seront floués, en colère, mais finalement contents.

Si l'intrigue est plus simple que celle de la plupart des comédies de Plaute et de Térence, il n'empêche qu'en humaniste, Piccolomini est grandement redevable à la comédie latine. On vient de voir que les personnages, et en particulier les personnages féminins, «se définissent les uns par rapport aux autres, le plus souvent deux par deux ", exactement comme le remarque Florence Dupont à propos des personnages du Phormion de Térence ${ }^{6}$. Mais c'est aussi dans la reprise de types féminins que consiste le travail d'imitation. La tradition littéraire lui offre un matériau brut, le caractère des personnages, qui se traduit par ses paroles.

4. «Envers personne plus qu'envers nous Vénus n’est bienveillante. / À la différence des autres, nous n’avons pas d'épouse fixe / qu'il faut garder, même si elle est acariâtre. / Jour après jour ce sont de nouvelles unions, de nouvelles noces / que nous célébrons : un amour nous plaît? nous retournons sur nos pas. / Il nous déplaît? nous changeons de route."

5. "[...] Mais comment penses-tu / que nous passerons la nuit? Pendant que tu besogneras la tienne / moi, je tiendrai la mienne contre ma poitrine, / je lui donnerai mes lèvres, la mordrai / et lui peloterai mille fois ses seins de neige! »

6. Florence Dupont, "La Psychologie des adulescentes dans l'action du Phormion de Térence ", REL, I986, t. 64 , p. 59 . 


\section{Les modèles féminins}

\section{Chrysis et Pythias}

Jean-Louis Charlet a déjà remarqué que les femmes présentes sur scène " appartiennent au monde de la comédie antique ${ }^{7}$ ". Ainsi, le personnage éponyme est déjà une prostituée dans l'Andrienne de Térence. À la différence de Piccolomini cependant, le dramaturge latin la dotait d'un passé. Mais loin de constituer une réelle épaisseur psychologique, ce rappel des faits avait pour fonction de faire comprendre au spectateur la situation présente. En effet, chez Térence, Chrysis n'est jamais visible, pour la bonne raison qu'elle est morte au moment où se noue l'action. Simon conte son histoire pour expliquer à Sosie l'attitude de son fils Pamphile. Deux explications rendent compte de ce choix opéré par Piccolomini. D'abord, on ne saurait trouver meilleur nom pour une prostituée. Chez Térence, venue d'Andros, elle quittait un travail pauvre et honnête, le filage de la laine, pour la vente de ses charmes. À cet égard, son nom est tout un programme : il fait entendre la note de l'or, et aussi celle de la chair. On sait que Piccolomini a appris le grec, à Florence, sous la férule de Francesco Filelfo. En grec, une chrysis est un vase en or : on retrouve ainsi la métaphore grivoise utilisée par Pythias et Lybiphanes. Mais à la sexualité s'ajoute l'idée de la vénalité. Ensuite, chez Térence, le personnage était déjà doté d'une sœur, Glycère, qui ne se prostituait pas plus que Pythias dans la comédie humaniste. Comme pour cette dernière, la relation qu' elle entretient avec son amant est désintéressée. Il faut toutefois nuancer en ajoutant que Glycère est amoureuse, alors que Pythias n'est guidée que par le plaisir. De plus, elle est enceinte, alors que la sœur de Chrysis est déjà mère. Notons que Pythias est une femme adultère, et que, comme la critique l'a remarqué, ce type est absent de la comédie latine. C'est cependant dans l'Eunuque de Térence que Piccolomini va chercher ce nom. Elle y est la servante de la prostituée Thaïs. Si le dramaturge humaniste emprunte ce personnage, c'est moins à cause de son rôle dans la pièce latine, où elle n'est qu'une exécutante, que parce que, comme l'a remarqué J.-L. Charlet, le nom est pris en exemple dans l'Épitre aux Pisons qui mentionne l' "audax Pythias $^{8}$ ». Ce nom renvoie à la malice et à l'effronterie.

Le travail d'imitation ne relève donc pas de la simple reprise. Piccolomini brode sur un canevas que lui offre Térence.

7. Enea Silvio Piccolomini, Chrysis, ouvr. cité, p. 28.

8. Chrysis, ouvr. cité, p. 29; Horace, Épître aux Pisons, v. 237-238. 


\section{Cassina}

On peut faire la même observation à propos de Plaute. Piccolomini lui prend le nom du personnage-titre, Casina (avec un ou deux « $s »)$. Ce nom est lui aussi programmatique. En grec, kassa signifie «courtisane " et kasis « la sœur ». D'un point de vue professionnel, Cassina est bien la semblable, la sœur de Chrysis. Chez Plaute, elle est l'objet de l'action : un vieillard libidineux, son fils et deux esclaves veulent jouir de ses faveurs. Chez Piccolomini, elle est aussi objet de l'action, comme on l'a déjà vu. Mais la Cassina antique est esclave, et non pas prostituée : "dans la comédie, elle ne f[ait] rien qui blesse la pudeur ", comme le dit le Prologue (v. 82-83), et elle reste pure jusqu'à ce que l'on apprenne qu'elle est la fille d'un citoyen d'Athènes.

\section{Canthara}

En ce qui concerne ce personnage, ce n'est pas pour son rôle dans l'action des pièces où elle est mentionnée que Piccolomini la choisit. Dans l'Epidicus de Plaute (v. 567-569), elle est une servante à qui Périphane ordonne d'aller chercher sa fille Telestis. Chez Térence, où elle apparaît trois fois, elle est nourrice ${ }^{9}$. C'est encore le nom qui explique la promotion au rang de personnage important. On sait que chez les Grecs, le cantharos est un grand vase à deux anses. Piccolomini filerait ainsi la métaphore. Au vase que s'échangent Lybiphanes et Pythias, à l'image de réceptacle contenue dans le nom de Chrysis, s'ajoute donc le personnage bien nommé de la maquerelle, probablement ancienne prostituée. Le symbole sexuel est probable et la pièce respire bien une odeur de lupanar. Mais surtout, comme l'ont remarqué les commentateurs, il s'agit d'un vase à boire le vin. J.-L. Charlet donne comme équivalent moderne de ce nom «bonbonne ». Pour ma part, je proposerais « dame-jeanne ». En effet, cette grosse bouteille en verre se caractérise, comme une bonbonne, par sa forme rebondie, par sa grande contenance (jusqu'à cinquante litres) et ce substantif présente l'avantage de comporter un prénom féminin. En français du Xvi ${ }^{\mathrm{e}}$ siècle, une jane est une bouteille. Piccolomini insiste sur cette assimilation en faisant dire à la vieille femme qu'elle est " comme une jarre où l'on garde le vin de Chios » ("Quasi lagena sum ubi vinum solet chium / condi [...]", v. 209-210). Reprenant la tradition de la comédie latine, Piccolomini introduit un personnage d'ivrognesse. Dans l'Andrienne de Térence, la sage-femme Lesbie

9. Dans l'Heautontimoroumenos, l'Andrienne (v. 769) et les Adelphes. 
était déjà présentée comme " temulenta ${ }^{10}$ " (imbibée), mais le modèle de la vieille pocharde est à chercher dans le Curculio de Plaute. La reprise textuelle est manifeste : Piccolomini adopte le commentaire que fait Palinure à la suite du portrait de la portière Lééna, brossé par Phédrome : "Quasi tu lagæenam dicas, ubi uinum Chium / solet esse» (v. 78-79). Elle l'est encore dans la fantaisie verbale que Piccolomini fait sienne, lui reprenant ses adjectifs composés, dont certains sont des néologismes (v. 205-206) :

Non uereor uera loqui : sum multibiba, merobiba, non limphibiba aut medobiba ${ }^{\mathrm{II}} \ldots$

Les détails retenus sont réalistes : " arent fauces" (" mon gosier est desséché ") (v. 202), "Pulmone ni[]hil / est meo sictius?" ("Y a-t-il rien de plus sec que mon poumon?") (v. 202-203). Entre le modèle et le personnage de Piccolomini, la différence est ténue, elle réside essentiellement dans le passage de la troisième personne du singulier à la première. On le voit encore avec l'expression qui résume le personnage : "sum uinosissima" s'exclame Canthara (" Je suis ivrognissime ») (v. 2Io), quand Plaute faisait dire à Phédrome : "uinosissima est ». La drôlerie est renforcée par ce changement dans l'énonciation. En effet, il est rare qu'un personnage s'autodévalorise à ce point. On a vu que Canthara pèse peu dans la marche de l'action. Le pittoresque est sa raison d'être. Il s'agit de placer devant le spectateur, pour le réjouir, un personnage caricatural. À la suite de Plaute, Piccolomini présente un fantoche contrefait qui se réduit à une passion. Comme Lééna, Canthara est une allégorie de la boisson. La comédie offre des types et joue sur des topoi. En l'occurrence il s'agit de celui de l'amour et du vin, présent dans la poésie érotique. On sait que les ailes de Cupidon peuvent y être imbibées de vin, comme l'exprime par exemple Ovide dans son Art d'aimer (I, 230-233) :

\section{Saepe illic positi teneris adducta lacertis Purpureus Bacchi cornua pressit Amor : \\ Vinaque cum bibulas sparsere Cupidinis alas, Permanet et capto stat gravis ille loco ${ }^{12}$.}

Or le spectacle qu'offre Canthara est celui de la dégradation de ces deux plaisirs de la vie, qui sont rendus avec grâce par les poètes. La délicatesse

Io. Térence, Andria, v. 229.

II. "Je ne crains pas de dire la vérité : je suis une grande buveuse, / buveuse de vin pur, pas buveuse d'eau, pas buveuse d'hydromel..."

I2. «Là, souvent l'Amour aux joues empourprées / presse dans ses petits bras l'amphore de Bacchus. / Dès que ses ailes sont imbibées de vin, / Cupidon, appesanti, reste immobile à sa place. » 
fait place à la grossièreté des traits, des attitudes et des paroles. C'est une vieille maquerelle qui, en usant autrefois de ses charmes, les a usés, et elle est alcoolique. Autrement dit, le spectacle de la comédie est burlesque. La beauté et le charme féminin d'une part, la griserie procurée par le vin, d'autre part, ont cédé la place à la grimace, à la laideur et à l'intempérance propres à déclencher un gros rire. Le propos de Theobulus, lors de son premier échange verbal avec elle, peut être repris par le spectateur : Te rideo [je ris de toi] (v. 260). La noblesse a été remplacée par la trivialité. C'est le triomphe de la bassesse. À la caricature s'ajoute la parodie, en l'occurrence, encore une fois, celle de la poésie amoureuse. Canthara parle du vin comme d'un être aimé dont l'absence, même brève, lui pèse (v. 2I2-2I4) :

[...]. Nunc eius amor me cupidam

letificat. Vbi, ubi est? Euax, est me prope ${ }^{13}$.

Ajoutons que Piccolomini modernise son personnage tout en insistant sur l'appartenance à une tradition. S'il est question de vin vieux et de vin de Chios, d'autres breuvages sont mentionnés (v. 207-208) :

neque ceruisiam bibo neque siceram.

Theucris hec largior et Boemis pocula ${ }^{14}$.

Ce refus a une double fonction. Pour ce qui concerne le personnage proprement dit, il s'agit de montrer que, ivrognissime, elle préfère les boissons fortes. En outre, la mention de ces breuvages produit un effet de réel pour les spectateurs de Nuremberg, habitués à boire de la bière et toujours préoccupés du danger ottoman. Quelques contemporains trouvent droit de cité dans sa pièce. Ainsi, à la scène II, Piccolomnini place un trait anti-français dans la bouche de Canthara et, comme l'a remarqué J.-L. Charlet, il fait allusion à l'habitude allemande, " de manger poisson et viande ensemble [...] alors qu’à Rome ces marchés étaient séparés ${ }^{15}$ » (v. 492-496) :

cetera que uolo gallica mercor fide.

A pistore cum panem pecierim,

uel cum vinum posco ex cenopolio,

aut ex macello piscem, agnum uel edum,

uacua redeo nisi es dederim ${ }^{16}$.

I3. "Mon désir pour lui me rend gaie. / Où est-il? où est-il? Ah! Il est près de moi! "

I4. "Je ne bois pas de bière ni d'autre boisson fermentée. / Je les abandonne aux Turcs et aux gens de Bohème. "

I5. Chrysis, ouvr. cité, p. I22.

I6. «Pour le reste, j'ai ce que je veux en achetant à la française : / quand je demande une miche au boulanger, / quand je demande du vin au cabaret, / ou au marché du poisson, de l'agneau, du chevreau, / je m’en retourne à vide si je ne donne pas de sous." 
Canthara a pour première interlocutrice Antiphila, qu' elle apostrophe lors de son entrée en scène. Elle est la destinataire des propos relatifs au vin. Son nom est encore un programme, littéralement, elle est " celle qui aime en échange ", en échange d'argent bien sûr. Comme le nom de Chrysis, celui-ci correspond donc exactement à la profession de prostituée. Piccolomini va chercher ce personnage dans l'Heautontimoroumenos de Térence, mais il le détourne. En effet, dans la comédie latine, Antiphila est une virgo, aimée de Clinia, la prostituée de la pièce s'appellant Bachis. On peut donc considérer que c'est uniquement pour la signification du nom que le personnage est retenu. L'accent est mis sur sa nature de prostituée. Elle est réduite à sa fonction amoureuse par son amant Archimenides. Elle est " programmée » pour l'amour (v. 235-237) :

Sed ubi tu nunc es, libello Venereo que me citasti? Ecce, me sisto; contrane ades?

\section{ANTIPHILA}

Adsum; nam, si absim, ni[c]hil mi[c]hi recusem mali ${ }^{17}$.

\section{La misogynie}

Ce monde de la prostitution peint à la mode antique est bien entendu un monde de plaisir. De la peinture que fait Dyophanes de la vie de bamboche menée avec son compagnon, on a déjà dit qu'elle était séduisante en ce qu'elle permet d'échapper aux femmes légitimes, considérées comme acariâtres. À la suite de Juvénal $(6,43)$, Piccolomini considère le mariage comme un capistrum, une " muselière" : "Coniugis maritus est vile mancipium» ("Le mari est la petite propriété de son épouse ») (v. I47).

Mais les prostituées ne sont pas présentées avec davantage de bienveillance, même si elles offrent un visage souriant et savent donner de la joie, leur raison d'être. Il faut s'arrêter un instant sur la comédie qu'elles jouent à plusieurs reprises à l'intérieur de cette comédie. Ce jeu est d'ailleurs ce qui les différencie des épouses qui, elles, n'endossent jamais aucun rôle, mais livrent brutalement leurs réactions. Aucune distance n'existe entre ce qu'elles disent et ce qu'elles éprouvent. Au contraire, les meretrices sont constamment en représentation. Leurs répliques sont ainsi stéréotypées dans

17. "Mais où es-tu maintenant toi qui avec ton programme / d'amour, m’a excité? Me voici. Es-tu là? " ANTIPHILIA. "Je suis là, car si je manquais, aucun mal ne me serait épargné. " 
leur contenu, dans leur forme et dans leur ton. Les hommes ne s'y trompent pas, qui reprennent leur propos pour en dénoncer l'avidité. Ainsi, Theobulus se déclare contrarié (v. 25-34) et fait entendre leur chanson. Chez Giraudoux il y aura la chanson des épouses ${ }^{18}$ qui trompent leurs maris sans vergogne, chez Piccolomini il y a celle des prostituées qui, cyniquement, dépouillent totalement les hommes à qui elles vendent leurs services (v. 25-34) :

\author{
"Antiqua est palla mea; nouam \\ faxo ut habeam : quatuor mi[c]hi da minas. \\ Baltheum nullum est mi[c]hi etiam : adiuua ut habeam ». \\ Hec uox mi[c] hi semper sonat in auribus. \\ Talentum impertitus sum, sed quo plus do, magis \\ expetit. «Hoc indigeo, hoc prebe, hoc uolo. \\ Aes quidem nullum in crumena superest mea; \\ despoliandus est quisquis indigens mei \\ advenerit. Ligari amantis marsupium \\ nequit ». Nil satis est meretricibus malis ${ }^{19}$.
}

L'intérêt de cette tirade est non seulement de reproduire le dialogue avec le client, de donner à voir le petit manège du commerce de la chair, mais aussi d'exprimer la pensée des prostituées. C'est le propre de la satire de mettre bas les masques et de faire éclater la vérité. La prostituée joue à l'indigente pour exciter la pitié de son interlocuteur. Mais Piccolomini fait entendre une sorte de voix off qui permet au spectateur de comprendre leur ressort essentiel : une cupidité sans bornes qui s'accompagne d'une volonté destructrice ("despoliandus est quisquis indigens mei »). Theobulus imagine les pensées de Cassina et de Chrysis et formule la maxime sur laquelle repose tout leur comportement : "Ligari amantis marsupium / nequit». La lucidité caractérise ici le personnage masculin qui semble assumer son statut de client. On peut penser que cette comédie dans la comédie ne concerne que les vieux clercs, dépourvus d'attraits physiques.

Mais le jeune Sedulius, qui semble pourtant être l'amant de cœur, fait lui aussi preuve de clairvoyance et montre, dans son premier échange avec Chrysis, qu'il sait à quoi s'en tenir (v. 59-6I) :

I8. Giraudoux, Electre, II, 6.

19. " "Mon manteau est vieux; j'entends bien en avoir / un neuf : donne-moi quatre mines. / Je n'ai plus de ceinture : aide-moi à m’en procurer une." / Mes oreilles résonnent de ces chansons. / Je lui ai donné un talent. Mais plus je lui donne, / plus elle en demande. "Je manque de ceci, offre-moi ceci, je veux ceci. / Il n'y a plus une pièce dans ma bourse. / Ruine obligatoire pour tout homme qui a besoin / de moi et vient me voir! La bourse d'un amant ne peut rester liée.” / Pour ces méchantes gagneuses ce n’est jamais assez. » 


\section{CHRYSIS}

Hem! Dantes! Quasi me plurimi nunc habeant, quod tu solus et unu, uel...

\section{SEDULIUS}

Aha, "uel"! Scio:

nemo nisi ego te unus... uel centum alii ${ }^{20}$.

Les professions de foi sonnent faux. Autrement dit, la comédie est une comédie grinçante. À des degrés divers, les personnages masculins, par leurs propos, brossent un portrait peu flatteur des prostituées.

Dans les vers suivants (v. 67-80), Lybiphanes, qui a assisté à l'échange verbal, achève de décrire la réalité de Chrysis : la prostituée tend des pièges, embrasse comme une femme honnête et peut verser des larmes sur commande. Qualis artifex! semble-t-il dire. Son propos vise à détourner Sedulius de la fréquentation des femmes vénales. La réponse que lui fait le maître est intéressante en ce qu'elle met en avant la structure farcesque de la pièce : il s'agit de tromper les trompeuses. Il faut savoir que la femme vénale est une comédienne pour ne pas devenir un "micheton ", ce rôle étant dévolu aux deux vieux clercs (v. 84-87) :

\section{SEDULIUS}

Animo

gerundus mos est; lubet ridere cum ioco.

Scortis ut scortis utor : simulant, simulo;

fingo me captum nec sum; par pari refero ${ }^{21}$.

Le but que semble se proposer Piccolomini en écrivant la Chrysis est de mettre en garde les jeunes gens contre les fadaises ("nugae », v. 8I) débitées par les professionnelles pour les piller. La définition de la prostituée donnée par Lybiphanes dit tout : elle n'est pas une femme («non mulier, meretrix. ", v. 93). Mettant dans la bouche de Canthara les propos que Plaute faisait tenir à la vieille maquerelle Cléérète dans l'Asinaria (I, 2I5-224), Piccolomini achève de déshumaniser la prostituée, qui n'est qu'un piège à hommes (268-269):

Hec domus est area, cibus est meretrix; ego sum auceps ac uos estis aues ${ }^{22}$ :

20. CHRYSIS. "Ah! "Les bons clients"! Comme s'ils étaient plusieurs à m’avoir! / Alors que tu es le seul, qu'il n'y a que toi et..." - SEDULIUS. "Ah, ah "et ..." Je sais : / personne d'autre que moi, et cent autres."

2I. SEDULIUS. «Il faut agir ainsi. Il me plaît / de jouer des tours aux filles quand je vais / aux filles. Feinte pour feinte! / Je fais semblant d'être pris et je ne le suis pas. Je leur rends la pareille. "

22. "Ma maison est le terrain : l'appât c'est la gagneuse;/ moi, je suis l'oiseleur et vous, vous êtes les oiseaux. » 
Cette fonction apotropaïque de la comédie culmine lorsque Theobulus compose le portrait des prostituées, qui s'oppose au petit blason composé par lui-même au début de la comédie. Plus de seins de neige à caresser, mais un tableau repoussant, parce que dégradé. Les charmes apparents sont frelatés, derrière l'apparence séduisante se cache la laideur. La prostituée se réduit à la chair dans ce qu'elle a de plus bas (v. 302-303; 310-3I4) :
[...] uenient, squalide, sordide,
inmund,e fimo ac stercore fetide;
$[\ldots]$
Colluuies primitus omnis hominum
fexque populi debet saturarier.
Tum, culinarum peruncte abdomine
eruptatoque uino in nostros madide
migrabunt amplexus; $[\ldots]^{23}$

Certes, le personnage parle par dépit. Mais, ce faisant, il exprime une conception misogyne qui renvoie à la tradition ecclésiastique. On retrouve en effet ici l'image de la femme-cloaque véhiculée par saint Augustin selon Ptolémée de Lucques : "Les prostituées sont dans la cité ce qu’un cloaque est dans un palais ${ }^{24}$."

Le monde de la prostitution est donc présenté comme le milieu de l'artifice par excellence. Les sentiments n'y sont pas de mise. À cet égard, ce monde est comme un miroir grossissant de la vérité des rapports hommes-femmes et ce sont ces dernières qui sont le plus attaquées. À Pythias qui affirme qu'aucun mari n'est fidèle à son épouse (v. I32-I33), Lybiphanes répond par une déclaration d'une totale misogynie. S'il a affirmé précédemment que la prostituée n'est pas une femme, la femme, de toute façon, n'est pas un être raisonnable. Elle est gourmande d'amour, insatiable, parce qu'elle est gouvernée par son corps qui la renvoie à l'animalité. Particulièrement développé, son appétit sexuel demande à être satisfait en permanence (v. 133-I37) :

Nec ullam marito feminam!

Casta est quam nemo rogat, nemo stimulat.

Oculo pocius solo quam solo uiro contenta est mulier : indomitum est animal, nullis regendum frenis nullisque monitis ${ }^{25}$.

23. «Elles viendront, sales, malpropres et crasseuses, / avec des relents de fange et de fumier ; / [...] / D'abord elles doivent rassasier toute l'ordure / humaine et la lie de la plèbe. / Alors, toutes enduites du gras des cuisines, / et de vin vomi, elles se transporteront jusqu'à nous / pour se faire embrasser. »

24. Cité par Charles Chauvin, Les chrétiens et la prostitution, Paris, Cerf, 1983, p. 2I.

25. «Et la femme n'est pas fidèle à son mari. / La femme chaste, c'est celle que personne ne recherche, que personne ne provoque. / Une femme se contente d'avoir un seul œil / plutôt qu'un seul homme. La femme est un / animal sauvage que ne saurait contenir aucun frein, aucune interdiction. " 
À bien y regarder, l'épouse ne diffère guère de la prostituée, la vénalité mise à part. On retrouve chez Piccolomini la bipartition à l'œuvre dans la farce qui présente soit des garces, soit des mégères, le caractère acariâtre de ces dernières s'expliquant par le fait qu'elles ne sont plus désirées de leurs maris. Ou bien, leur manque de grâces explique qu'elles n'accèdent même pas au statut d'épouse.

En reprenant à la comédie antique des noms et des types de femmes, Piccolomini amuse son public. Mais cet amusement n'est pas gratuit, il ne s'agit pas de décalquer simplement des modèles. Certes le milieu de la prostitution est consubstantiel aux ouvres des dramaturges latins. De façon révélatrice, lorsque Trechsel, en I502, publiera le théâtre de Térence, il accompagnera le texte d'une gravure représentant un édifice théâtral où se trouve un lupanar. Il est donc naturel de constater que, dans la Chrysis, il est question de prostitution. Mais à la différence de ses modèles antiques, la pièce ne présente que le milieu de la prostitution. Toutes les femmes en font partie, et, lorsque ce n'est pas le cas, le personnage est infidèle. Dans l'univers féminin de la Chrysis, la femme honnête n'existe pas. Cette vision négative peut en partie s'expliquer par la biographie de l'auteur. La Chrysis correspond à la crise que traverse Piccolomini, fatigué de la vie libertine qu'il a menée jusqu'alors. Dans la préface de son édition, J.-L. Charlet rappelle que le De miseria curialium date du 30 novembre I444. Enea Silvio ne voit plus la splendeur des courtisanes, il n'en perçoit désormais que la misère. On a l'impression que c'est de la femme en général que se détourne le curiale. Si cette comédie offre de quoi réjouir le spectateur, le rire qu'elle produit est, à certains égards, un rire jaune. De ce point de vue, la vision de la femme qu'elle véhicule est en conformité avec celle d'une Église dont Piccolomini prendra la tête. À la suite de saint Paul, il invite à renoncer aux plaisirs de la chair : "[...] revêtez-vous du Seigneur Jésus-Christ et ne tenez pas compte de la chair en ses convoitises ${ }^{26}$. " On sait que ce verset a changé la vie d'Augustin. Le moment de rédaction de la Chrysis est celui d'une metanoia commençante, semblable, mutatis mutandis, à la fameuse scène du jardin en août 386, où Augustin a entendu une voix lui dire de lire saint Paul ${ }^{27}$. Pour se persuader du changement radical qui intervient dans l'existence de Piccolomini, il n'est que de se référer à la lettre écrite deux ans plus tard à Hippolyte de Milan (3 janvier I446) et connue sous le nom de De remedio amoris. Afin de dissuader son correspondant déjà âgé de continuer à aimer une prostituée « qui couche pour de l'argent avec

26. Paul, Épittre aux Romains, XIII, I4.

27. Augustin, Les Confessions, VIII, I2, 29. 
n'importe quel homme ${ }^{28} "$, il utilise Térence ${ }^{29}$, montre que la maladie d'amour frappe aussi bien les vieillards que les jeunes gens ${ }^{30}$, et donne de la femme une définition qui s'applique en tout point aux créatures de la Chrysis : "Qu'est-ce qu'une femme, je te prie, sinon une voleuse de jeunesse, une détrousseuse d'hommes, la mort des vieillards, la dissipatrice du patrimoine, la ruine des honneurs, l'aliment du diable, la porte de la mort et l'auxiliaire de l'enfer ${ }^{31}$ ? " Dans la Lettre à Mariano Sozzini, qui contient l'Historia de duobus amantibus, et qui est exactement contemporaine de la Chrysis, Piccolomini insiste sur son âge (quarante ans) et sur celui de son correspondant (cinquante ans) : à l'automne de la vie, il est inconvenant de vouloir aimer. Autant dire que s'ils se laissent prendre à l'amour, et en particulier à l'amour vénal, ils seront des Theobulus et Dyophanes. Pour formuler la leçon qui se dégage de la pièce, on peut reprendre l'injonction qu'au siècle suivant formulera en France Pierre Grosnet dans son Haud inutile libidinis siue luxuriae dehortamentum : "fugite fornicationem! " La Chrysis est elle aussi un dehortamentum qui invite à fuir les femmes.

28. E. S. Piccolomini, Euvres érotiques, trad. de Frédéric Duval, Turnhout, Brepols, 2003, p. 217.

29. Ibid., p. 219: «De là vient cette réplique de Parménion chez Térence : "Dieux bons, quelle est cette maladie? Les hommes sont-ils tellement changés par l'amour qu'on ne puisse plus les reconnaître?" " [Eunuque, 225]

30. Ibid., p. 219 : « [...] la luxure [...] est une sorte de maladie redoutable qui attaque la plupart des jeunes gens, mais s'en prend aussi aux hommes mûrs et aux vieillards. Plus la personne qui en est la proie est censée être âgée ou savante, plus cette maladie est dangereuse et risible. "

3I. Ibid., p. 223. 